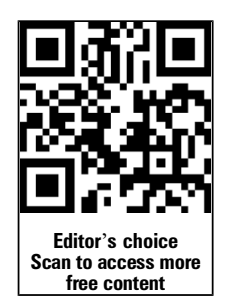

${ }^{1}$ Department of Immunology, North Bristol NHS Trust, Bristol, UK

${ }^{2}$ Department of Cellular Pathology, North Bristol NHS Trust, Bristol, UK

\section{Correspondence to} Dr Philip Bright, Immunology Department, North Bristol NHS Trust, Pathology Sciences Building, Southmead Hospital, Southmead, Bristol BS10 5NB, UK; philip.bright@doctors.org.uk

Received 15 August 2014 Revised 2 October 2014 Accepted 9 October 2014 Published Online First 28 October 2014

\section{CrossMark}

\title{
Laboratory clues to immunodeficiency; missed chances for early diagnosis?
}

\author{
P D Bright, ${ }^{1}$ N Rooney, ${ }^{2}$ P F Virgo, ${ }^{1}$ R J Lock, ${ }^{1}$ S L Johnston, ${ }^{1}$ D J Unsworth ${ }^{1}$
}

\section{ABSTRACT}

Primary immunodeficiency is seen in an estimated one in 1200 people, and secondary immunodeficiency is increasingly common, particularly with the use of immunosuppresion, cancer therapies and the newer biological therapies such as rituximab. Delays in the diagnosis of immunodeficiency predictably lead to preventable organ damage. Examples of abnormal pathology tests that suggest immunodeficiency from all laboratory specialities are given, where vigilant interpretation of abnormal results may prompt earlier diagnosis. If immunodeficiency is suspected, suggested directed testing could include measuring immunoglobulins, a lymphocyte count and T-cell and B-cell subsets.

\section{INTRODUCTION}

The prevalence of primary immunodeficiency (PID) has been estimated at one in 1200 people based on telephone surveys in the USA. ${ }^{1}$ Antibody deficiency is the diagnosis in approximately $65 \%$ of those with PID. ${ }^{2}$ Significantly, there are often delays of many years ${ }^{3}$ in the diagnosis of PID, and late diagnosis increases the risk of chronic organ damage (eg, correlation between delayed diagnosis of X-linked agammaglobulinaemia and bronchiectasis ${ }^{4}$ ). Infection frequency ${ }^{5-7}$ and the directly resultant organ damage ${ }^{8}$ can be prevented through appropriate treatment, such as lifelong prophylaxis using repeated infusions of donor-pooled human immunoglobulin G. ${ }^{9}$

Often, but not always, PID presents with recurrent infection with familiar pathogens and/or infection with opportunist or atypical organisms (eg, Pneumocystis jerovici, giardiasis, atypical mycobacteria). Paradoxically, in addition to risk of infection, PID can also increase the risk of autoimmune diseases, such as immune thrombocytopenic purpura (ITP), presumably related to immune dysregulation.

Pathology tests undertaken for other reasons may unexpectedly generate abnormal laboratory results indicative of possible immunodeficiency. It is important that pathology laboratory staff across all disciplines consider primary and secondary immunodeficiency as a possible explanation in these cases and this be highlighted on laboratory test reports for requesting clinicians. This can lead to earlier diagnosis and treatment, and improved outcome for patients. We now present a series of examples of abnormal laboratory results indicative of possible immune deficiency listed by laboratory discipline.

\section{Biochemistry testing}

- Low immunoglobulins: Testing of immunoglobulins can reveal low levels of $\operatorname{IgM}, \operatorname{IgA}$ and/or
IgG, which may be primary or secondary, and should not be ignored. The significance of a selective low $\operatorname{IgM}$ is unclear although it can be associated to ageing, autoimmunity and lymphoproliferative disease. ${ }^{10}$ A selective low IgA is found in many apparently healthy people although may be associated with autoimmunity, allergy and an increased frequency of infections. ${ }^{11-13}$ A low IgG may well represent significant immunodeficiency, especially when associated to a history of unusual or recurrent respiratory or gastrointestinal infections. Reference ranges should be age appropriate, particularly in early life when transferred maternal $\mathrm{IgG}$ will wane, and endogenous production of immunoglobulin may not yet have matured.

- High immunoglobulins: High immunoglobulin levels paradoxically may also point to significant immunodeficiency. In a 1-year period in our centre, there were 6627 tests measuring IgG, of which 203 were above $30 \mathrm{~g} / \mathrm{L}$ (in 79 patients). Of these 79 patients, 62 had monoclonal paraproteins and 17 had polyclonal increases in globulins. Of these 17 patients, one patient had a new diagnosis of HIV (which is often associated with gross hypergammaglobulinaemia), four had autoimmune hepatitis/primary biliary cirrhosis, two had Sjögren's syndrome, three were likely infection related and seven had unclear diagnoses. High levels of polyclonal $\mathrm{IgG}$ are often reported as reactive, but in our clinical experience, very high polyclonal levels (eg, IgG $>30 \mathrm{~g} / \mathrm{L}$ ) should prompt specific consideration of HIV infection.

- Coeliac disease serology: Specific IgA deficiency is usually a benign disease and is relatively common with a prevalence of between 1 in 223 and 1 in $1000,{ }^{14}$ but with a higher prevalence (eg, 1 in 39) in coeliac disease. ${ }^{15}$ Specific IgA deficiency may be diagnosed following serological testing for coeliac disease by the finding of a low background IgA concentration, and a low or absent IgA may result in a falsely negative result on IgA-based coeliac assays (eg, IgA antitissue transglutaminase assay (IgA TTG)) necessitating additional testing by less sensitive and specific IgG-based assays. Low background optical density on the IgA TTG assay or a low background staining on antiendomysial IgA testing $^{16} 17$ should lead to the suspicion of IgA deficiency. In our laboratory, 1 in 190 routine samples have a low background IgA TTG (unpublished data using an optical density cut-off of $<0.06$ on the Quantalite Rh tTG IgA ELISA, INOVA Diagnostics, San Diego) suggestive of IgA deficiency, and we would therefore 
measure their IgA concentration. Some IgA-deficient patients will also have deficiencies of other immunoglobulin classes, and we therefore advocate checking levels of IgM and IgG in those with confirmed IgA deficiency. We have diagnosed several patients with pan-hypogammaglobulinaemia via this route of laboratory testing. ${ }^{18}$

- Total IgE: Similarly to a low IgA, a very low or absent total IgE level can be an incidental finding when investigating patients for possible allergy. Very low total IgE (eg, $<2$ international units) may represent antibody deficiency in an estimated $7 \%$ of those with a very low IgE. ${ }^{19}$ Screening of these samples by testing of other immunoglobulin isotypes is worth consideration and has led to new diagnoses of significant antibody deficiency. ${ }^{19}$

- Serum-free light chains (SFLC): SFLCs are tested in the investigation of possible B-cell clonality and amyloidosis. Abnormal levels or ratios of $\kappa / \lambda$ light chains are normally the result of increased clonal production of one or other of the light chains and suggest B-cell malignancy. However, PID involving B-cell dysfunction may also result in non-clonal abnormal ratios of $\kappa / \lambda$ light chains with levels of light chains typically very low. ${ }^{20}$

- Serum globulin gap: Serum globulin forms approximately $40 \%$ of total immunoglobulins. ${ }^{21}$ The globulin gap is an estimate of the serum globulin value and can be calculated by subtracting the serum albumin from the serum protein. The globulin fraction can be reduced in antibody deficiency because immunoglobulins make a major contribution to the total globulin value. Follow-on measurement of IgG concentration for patients found to have a low globulin gap uncovered many patients with a low IgG concentration (eg, 89\% of patients with a globulin gap $<18 \mathrm{~g} / \mathrm{L}$ had an $\operatorname{IgG}<6 \mathrm{~g} / \mathrm{L}){ }^{22}$

- Serum protein electrophoresis (SPE): SPE is primarily a test looking for clonal production of antibody in the investigation of suspected multiple myeloma and is performed without testing immunoglobulin $\mathrm{A}, \mathrm{G}$ and $\mathrm{M}$ levels in some centres. As well as increased levels of globulins, SPE may reveal a reduced gamma globulin region (hypogammaglobulinaemia) indicating possible antibody deficiency or, indeed, a lightchain clonal disorder (which is often not detected on SPE)see figure 1 below. A reduced gamma globulin zone on SPE should prompt an immunofixation for a light-chain clonal disorder, and measurement of $\operatorname{IgG}, \operatorname{IgA}$ and $\operatorname{IgM}$ to make the diagnosis of antibody deficiency.

\section{Haematology testing}

- Lymphocyte count: Persistent unexplained lymphocytopenia is often ignored, but may be a key clue suggesting PID ${ }^{23}$ particularly in the first few months of life. Severe Combined Immune Deficiency (SCID) often leads to pan- lymphocytopenia, but selective deficiencies in one or other subset (eg, T-cell deficiency or selective CD4 T-cell deficiency) can be masked within a normal total count. A low lymphocyte count may be a primary or secondary phenomenon and may indicate significant immune suppression (including HIV infection) and should prompt investigation of the underlying cause. If a cause cannot be ascertained then consideration should be given to measuring T/B-cell lymphocyte subsets to exclude SCID (especially in young children) and immunoglobulins. In the first few months of life, IgG levels are a result of transferred maternal IgG in the late stages of pregnancy. It should be noted that the reference ranges of lymphocytes should be age appropriate and in babies/infants, are significantly higher than in adults. ${ }^{24}$ The unwary may unwittingly interpret a lymphocyte count as normal in an infant as it is within the normal adult reference range, when it is, in fact, very low for age.

- Monocyte count: Monocytopenia has recently been recognised within a new PID caused by GATA-2 deficiency ${ }^{25}$ involving a predisposition to human papilloma virus and/or atypical mycobacterial infection, and high risk of myelodysplasia and acute myeloid leukaemia. Although this condition is likely extremely rare, a persistently absent or very low monocyte count may indicate this condition and should not be ignored.

- Platelet count: ITP has an estimated incidence of 2 in 100000 adults $^{26}$ and can be a presenting feature of primary $^{27}$ or secondary ${ }^{28}$ immunodeficiency. Similarly, autoimmune haemolytic anaemia and autoimmune neutropenia can be presenting features of immunodeficiency. ${ }^{23}$ In addition to considering lupus and other plausible autoimmune pathologies for cytopenias, HIV and PID diagnoses, such as common variable immune deficiency (CVID) should also enter the differential. CVID is the most common clinically significant PID with a prevalence of around 1 in $25000 .^{29}$ ITP was present in $6 \%$ of patients with CVID in one study. ${ }^{30}$ Quantification of serum immunoglobulins in cases of ITP is advised in published guidance ${ }^{31}$ to exclude antibody deficiencies such as CVID.

- Platelet volume: Platelet volume is measured in the normal processing of a full blood count but is usually not reported. Individual low platelet volume is strongly suggestive of Wiskott-Aldrich syndrome. Although this syndrome is extremely rare and nearly always in male children (eg, 1-10 cases per million live births ${ }^{32}$ ), the finding of a low platelet size should prompt consideration of this diagnosis.

\section{Blood transfusion testing}

- Reverse cross-match: Routine erythrocyte cross-matching is based on serological techniques (haemagglutination) using monoclonal antibodies to determine $\mathrm{ABO}$ blood type. A
Figure 1 Serum (capillary zone) protein electrophoresis of a normal control (A) showing albumin, $\alpha 1, \alpha 2$, $\beta 1, \beta 2$ and $\gamma$ peaks, and from a patient (B) with hypogammaglobulinaemia with a reduced $\gamma$ peak.
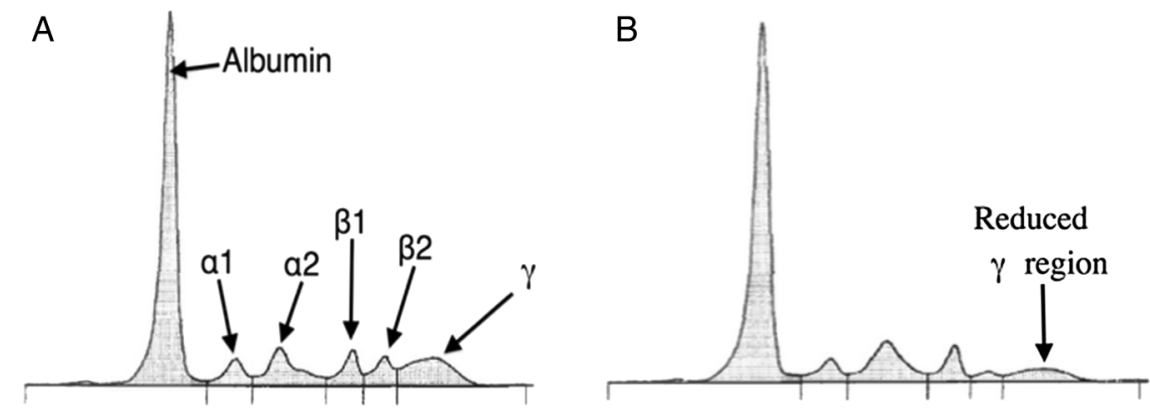
Figure 2 Large bowel biopsy in a patient with chronic diarrhoea with severe primary T-cell immunodeficiency showing: (A) H\&E stain of endothelial cell nuclear inclusions of cytomegalovirus (CMV) (circled). (B) CMV specific staining (brown and arrowed).
A

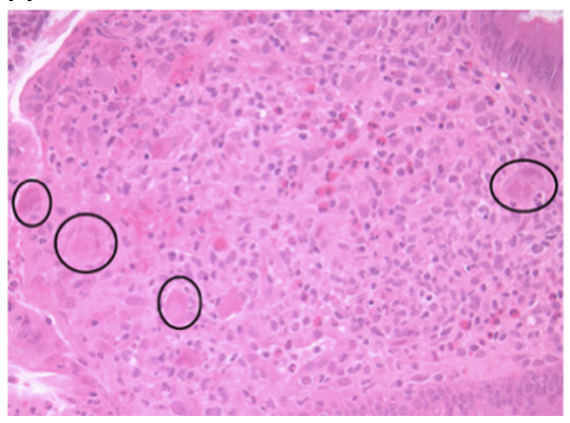

B

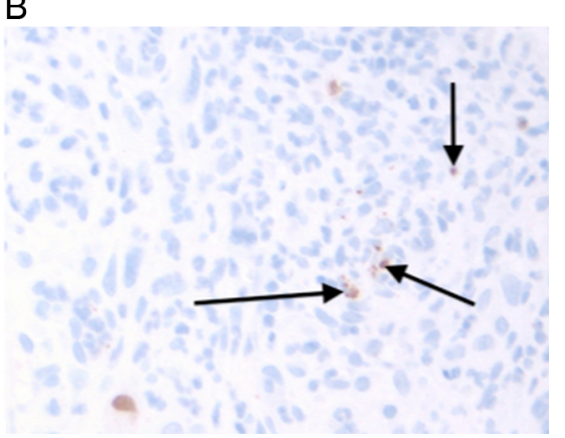

confirmatory 'reverse group' may be performed by adding patient serum to a defined panel of donor red cells with known $\mathrm{ABO}$ blood group. Patient serum should contain 'natural' anti-A and anti-B antibodies (also called isoagglutinins) in predictable patterns which correlate to the cell group (eg, patient with blood group A will have natural B antibodies, but no natural A antibodies). Screening by this method will detect IgM antibodies preferentially (IgG antibodies are poor agglutinators of red cells). In the presence of antibody immunodeficiency, this 'reverse group' may fail, indicating a lack of anti-A/B IgM type antibodies. This is commonly found in the young (as babies do not make natural $\mathrm{A}$ and $\mathrm{B}$ antibodies until approximately 3 months of age) and the old, and may indicate significant and treatable immunodeficiency in some situations. IgM levels do normally fall in old age, ${ }^{33}$ but not to the extent they are undetectable. Consideration should be given to quantifying immunoglobulins in patients (over the age of 3 months) if they have an unexpected non-reactive reverse group. However, many patients such as those in oncology or haematology clinics may well have an expected failure of the 'reverse group'.

\section{Genetics testing}

- Cytogenetics testing: This test is often performed in infants with failure to thrive or babies with syndromic features looking for chromosomal abnormalities, although it is starting to be replaced by other test modalities. The cytogenetics test involves non-antigen-specific mitogenic (eg, phytohaemagglutinin) stimulation of lymphocytes, causing quiescent cells to undergo mitosis, so metaphase chromosomes can be obtained for analysis. These metaphase chromosomes are then easier to visualise. A failed test may indicate a numerical or functional T-cell deficiency. A real possibility here is the diagnosis of SCID, where delayed diagnosis, and failure to proceed to early bone marrow transplant may unfortunately lead to life-threatening infections (eg, Epstein-Barr virus (EBV), cytomegalovirus (CMV), or disseminated lifethreatening infection from normally benign live vaccines including polio or Bacillus Calmette-Guerin). A failed test should prompt consideration of repeat cytogenetics testing with parallel lymphocyte subset analysis. ${ }^{34}$

\section{Microbiology/virology testing}

- Specific vaccine responses: Patients with PID respond poorly to routine vaccinations. When these vaccines contain live organisms, they carry significant risk of disease to patients with PID. Failed vaccine responses, especially when global, are suggestive of antibody immunodeficiency. A clinical episode of measles in a patient previously fully vaccinated, seronegativity in a patient previously vaccinated showing a failure of vaccine response (although failure to respond to Hepatitis $B$ vaccination is found in $10 \%-15 \%$ of the popula$\operatorname{tion}^{35}$ ) or negative varicella zoster virus or EBV antibody results in adults should raise suspicion. Primary vaccine failure rates are thought to be relatively low for Rubella $(<8 \%)^{36}$ for example, so seronegativity in pregnant women who have been vaccinated may be another presentation of immunodeficiency and should trigger consideration of quantifying immunoglobulins and lymphocyte subsets.

- Unusual/recurrent positive cultures: Certain infections are associated with particular immunodeficiencies. ${ }^{2} 37$ For example:

- Antibody deficiencies:

$\bigcirc$ Recurrent infection of the upper and lower respiratory tract with encapsulated organisms such as Streptococcus pneumoniae and Haemophilus influenzae.

O Gastrointestinal infection with Giardia lamblia and Campylobacter jejuni.

- T-cell deficiencies:

O Recurrent or severe candida infection.

O Pneumocystis jiroveci pneumonia.

O Unexpected, recurrent or severe viral infections such as human papilloma virus or CMV (see figure 2 of a biopsy unexpectedly showing CMV)

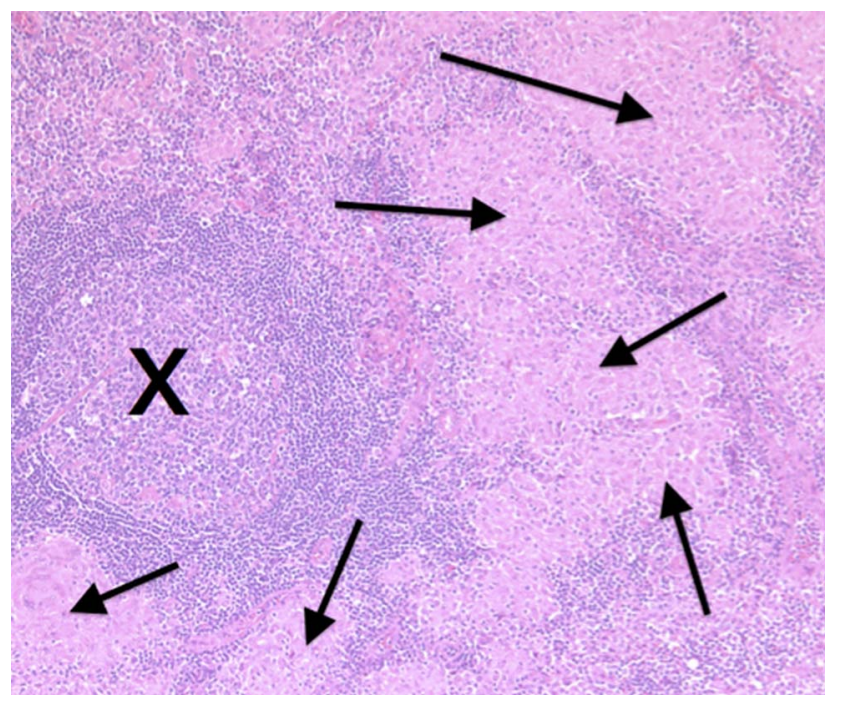

Figure $3 \mathrm{H} \& \mathrm{E}$ stain of a biopsy from the axillary node of a patient with granulomatous common variable immune deficiency showing a germinal centre (marked $\mathrm{X}$ ) and multiple granulomas (arrowed). 


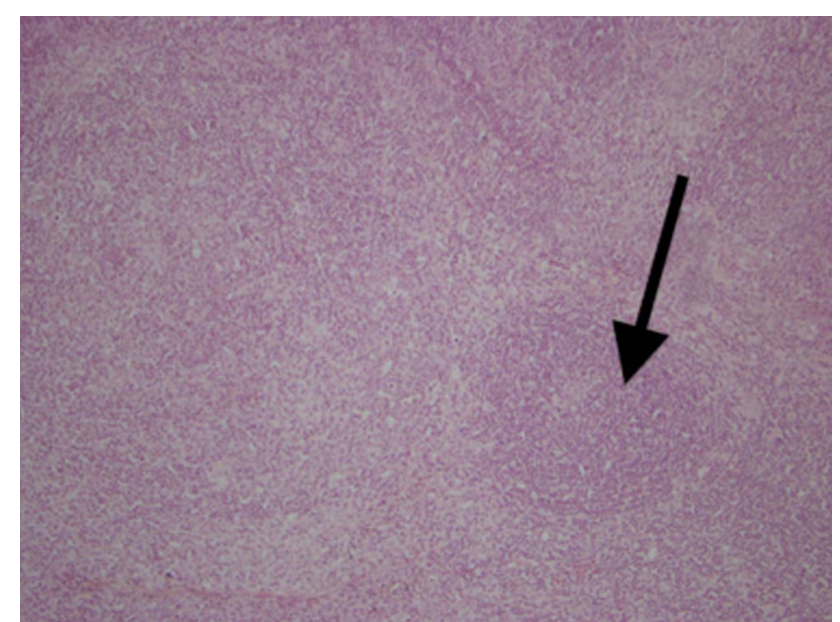

Figure 4 H\&E stain of tonsillar tissue from a paediatric patient with hyperimmunoglobulin $M$ syndrome showing lymphoid aggregates (arrowed) without the formation of an active germinal centre. The appearances could be mistaken for nodular lymphocyte predominant Hodgkin lymphoma.

- Complement deficiencies (very rare):

$\bigcirc$ Recurrent infection with Neisseria meningitides (often unusual serotypes) is associated with deficiencies of the common terminal complement pathway, and can be managed by vaccination and prophylactic antibiotics, with consideration for screening of relatives.

\section{Histopathology testing}

There are multiple characteristics of PIDs that may be found on histopathological biopsy inspection. A few examples are given below in addition to the case in figure 2:
- Granulomata may be found in conditions such as Mycobacterium tuberculosis, sarcoidosis or Crohn's disease, but may also be found in the granulomatous variant of common variable immunodeficiency (see figure 3). An unexpected finding of granulomata, including all cases of 'sarcoid', should prompt consideration for the measurement of immunoglobulins. In sarcoidosis the immunoglobulins would be expected to be raised, rather than in granulomatous CVID, where reduced immunoglobulins would be expected.

- Normal lymphoid tissue will contain active germinal centres with a light and dark zone, but these may be absent or abnormal in immunodeficiency syndromes like hyper-IgM syndrome, where mutations in one of a number of different genes prevent expected T-cell and B-cell interaction. Absent germinal centres on lymph node biopsy should prompt measurement of immunoglobulins and lymphocyte subsets (see figure 4).

- The finding of villous atrophy on small bowel biopsy is consistent with a diagnosis of coeliac disease. However, villous shortening may be associated with infection such as Giardia lamblia ${ }^{38}$ which is a common infection found in antibody deficiency.

- The absence of plasma cells in biopsies may also be the result of certain PIDs where B-cells are low or absent, such as X-linked agammaglobulinaemia (see figure 5), autosomal recessive agammaglobulinaemia, or a subset of patients with common variable immunodeficiency.

\section{CONCLUSION}

Immunodeficiency may be unfamiliar, but is not that rare, and may be primary or secondary. The most common primary immunodeficiencies are antibody deficiencies ${ }^{39}$ of which the predominant form is common variable immunodeficiency. The incidence of secondary immunodeficiency is increasing, particularly with the use of biologically targeted immunosuppressive

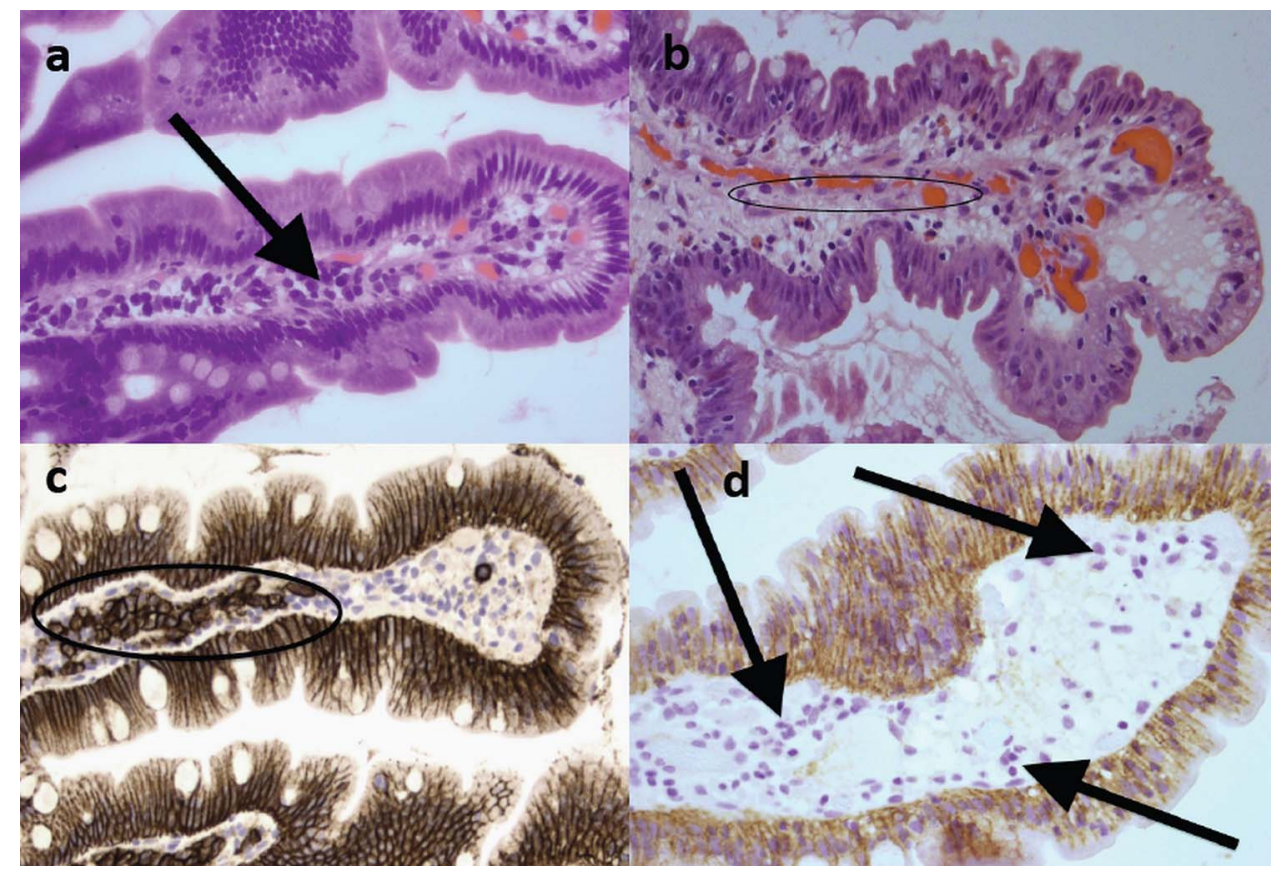

Figure 5 H\&E stains of the small bowel of: (A) A normal patient where plasma cells are evident in the lamina propria of the biopsy (arrowed). (B) A patient with X-linked agammaglobulinaemia (XLA) where plasma cells are lacking in the lamina propria (flattened circle). Brown plasma cell staining (plasma cell surface marker CD138) of the duodenum of: (C) A control patient with normal expression of plasma cells in the lamina propria (circled). (D) A patient with XLA showing absent plasma cells in the lamina propria. Lymphocytes in the lamina propria do not stain with CD138 and are therefore T-cells (arrowed). 
drugs such as rituximab (which targets CD20 expressing B-cells and can cause low antibodies). ${ }^{40}{ }^{41}$ Rituximab can aggravate pre-existing antibody deficiency. ${ }^{42}{ }^{43}$ SCID has an approximate incidence of 1 in 50000 live births. ${ }^{44}$ The consequences of delayed diagnosis of immunodeficiency can be recurrent, severe and potentially life-threatening infections and/or chronic organ damage (eg, bronchiectasis). Pathology tests are performed on many of these patients prior to their diagnosis of immunodeficiency, and offer an opportunity for earlier diagnosis and appropriate intervention to prevent morbidity and mortality, highlighting the need for appropriate correlation and interpretation. Actioning abnormal pathology tests may involve reflex testing (eg, immunoglobulins for low IgA on coeliac testing), reflex automated comments (eg, please consider immunodeficiency), tailored comments or direct discussion with clinical colleagues as to the need for further investigation.

Contributors DJU conceived the idea for the paper, PDB wrote the manuscript, PFV/DJU/RJL/SLJ were involved in finding example cases for the publication, NR provided interpretation of histopathological interpretation and the histopathological slides. All authors read, edited and approved the final manuscript.

\section{Competing interests None.}

Provenance and peer review Not commissioned; externally peer reviewed.

\section{REFERENCES}

1 Boyle JM, Buckley RH. Population prevalence of diagnosed primary immunodeficiency diseases in the United States. J Clin Immunol 2007;27:497-502.

2 Turvey SE, Bonilla FA, Junker AK. Primary immunodeficiency diseases: a practical guide for clinicians. Postgrad Med J 2009;85:660-6.

3 Thickett KM, Kumararatne DS, Banerjee AK, et al. Common variable immune deficiency: respiratory manifestations, pulmonary function and high-resolution CT scan findings. QJM 2002;95:655-62.

4 Plebani A, Soresina A, Rondelli R, et al. Clinical, immunological, and molecular analysis in a large cohort of patients with $x$-linked agammaglobulinemia: an Italian multicenter study. Clin Immunol 2002;104:221-30.

5 Busse PJ, Razvi S, Cunningham-Rundles C. Efficacy of intravenous immunoglobulin in the prevention of pneumonia in patients with common variable immunodeficiency. J Allergy Clin Immunol 2002;109:1001-4.

6 Cunningham-Rundles C, Siegal FP, Smithwick EM, et al. Efficacy of intravenous immunoglobulin in primary humoral immunodeficiency disease. Ann Intern Med 1984:101:435-9.

7 Quartier P, Debre M, De Blic J, et al. Early and prolonged intravenous immunoglobulin replacement therapy in childhood agammaglobulinemia: a retrospective survey of 31 patients. J Pediatr 1999;134:589-96.

8 De Gracia J, Vendrell M, Alvarez A, et al. Immunoglobulin therapy to control lung damage in patients with common variable immunodeficiency. Int Immunopharmacol 2004;4:745-53.

9 Stadtmauer G, Cunningham-Rundles C. Outcome analysis and cost assessment in immunologic disorders. JAMA 1997;278:2018-23.

10 Hobbs JR. IgM deficiency. Birth Defects Orig Artic Ser 1975;11:112-16.

11 Latiff AHA, Kerr MA. The clinical significance of immunoglobulin A deficiency. Ann Clin Biochem 2007:44:131-9.

12 Aittoniemi J, Koskinen S, Laippala P, et al. The significance of IgG subclasses and mannan-binding lectin (MBL) for susceptibility to infection in apparently healthy adults with IgA deficiency. Clin Exp Immunol 1999;116:505-8.

13 Kaufman HS, Bobbs JR. Immunoglobulin deficiencies in an atopic population. Lancet 1970;2:1061-3.

14 Cunningham-Rundles C. Physiology of IgA and IgA deficiency. I Clin Immunol 2001;21:303-9.

15 Wang $\mathrm{N}$, Shen $\mathrm{N}$, Vyse TJ, et al. Selective IgA deficiency in autoimmune diseases. Mol Med 2011:17:1383-96.

16 Sinclair D, Saas M, Turk $A$, et al. Do we need to measure total serum IgA to exclude IgA deficiency in coeliac disease? I Clin Pathol 2006;59:736-9.
17 NICE Clinical Guideline 86. Coeliac Disease; Recognition and Assessment of Coeliac Disease. 2009. http://www.nice.org.uk (accessed 4 Aug 2014).

18 Bright P, Lock RJ, Unsworth DJ. Immunoglobulin A deficiency on serological coeliac screening: an opportunity for early diagnosis of hypogammaglobulinaemia. Ann Clin Biochem 2012;49(Pt 5):503-4.

19 Unsworth DJ, Virgo PF, Lock RJ. Immunoglobulin E deficiency: a forgotten clue pointing to possible immunodeficiency? Ann Clin Biochem 2011;48(Pt 5):459-61.

20 Unsworth DJ, Wallage MJ, Sarkar $E$, et al. Abnormalities of serum-free light chain in patients with primary antibody deficiency in the absence of B lymphocyte clonality. J Clin Pathol 2012;65:1128-31.

21 Burtis CA, Ashwood ER. Tietz textbook of clinical chemistry. 2nd edn, Philadelphia: WB Saunders Company, 1994.

22 Jolles $\mathrm{S}$, Borrell R, Zouqail $\mathrm{S}$, et al. Calculated globulin (CG) as a screening test for antibody deficiency. Clin Exp Immunol 2104;177:671-8.

23 Krishna MT, Tarrant IL, Cheadle EA, et al. An audit of lymphopenia in infants under 3months of age. Arch Dis Child 2008;93:90-1.

24 Hannet I, Erkeller-Yuksel F, Lydyard P, et al. Developmental and maturational changes in human blood lymphocyte subpopulations. Immunol Today 1992;13:215, 218.

25 Hsu AP, Sampaio EP, Khan J, et al. Mutations in GATA2 are associated with the autosomal dominant and sporadic monocytopenia and mycobacterial infection (MonoMAC) syndrome. Blood 2011;118:2653-5.

26 Fogarty PF, Segal JB. The epidemiology of immune thrombocytopenic purpura. Curr Opin Hematol 2007; 14:515-19.

27 Podjasek JC, Abraham RS. Autoimmune cytopenias in common variable immunodeficiency. Front Immunol 2012;3:189.

28 Glatt AE, Anand A. Thrombocytopenia in patients infected with human immunodeficiency virus: treatment update. Clin Infect Dis 1995;21:415-23.

29 Hammarström L, Vorechovsky I, Webster D. Selective IgA deficiency (SIgAD) and common variable immunodeficiency (CVID). Clin Exp Immunol 2000;120:225-31.

30 Cunningham-Rundles C, Bodian C. Common variable immunodeficiency: clinical and immunological features of 248 patients. Clin Immunol 1999;92:34-48.

31 Provan D, Stasi R, Newland AC, et al. International consensus report on the investigation and management of primary immune thrombocytopenia. Blood 2010;115:168-86.

32 Ochs HD, Thrasher AJ. The Wiskott-Aldrich syndrome. J Allergy Clin Immunol 2006;117:725-38.

33 Lock RJ, Unsworth DJ. Immunoglobulins and immunoglobulin subclasses in the elderly. Annals Clin Biochem 2003;40:143-8.

34 Unsworth DJ, Thomas HM. Missed clues and delayed diagnosis of immunodeficiency. Lancet 1997;349:435.

35 Salisbury D, Ramsay M, Noakes K. Green book: immunisation against infectious disease. 2006. http://webarchive.nationalarchives.gov.uk/20080910134953/http:// dh.gov.uk/en/Publicationsandstatistics/Publications/PublicationsPolicyAndGuidance/ DH_079917 (accessed 4 Aug 2014).

36 Singh $\mathrm{R}$, John TJ, Cherian T, et al. Immune response to measles, mumps \& rubella vaccine at 9, 12 \& 15 months of age. Indian J Med Res 1994;100:155-9.

37 Azar AE, Ballas ZK. Evaluation of the adult with suspected immunodeficiency. Am J Med 2007:120:764-8.

38 Oberhuber G, Kastner N, Stolte M. Giardiasis: a histologic analysis of 567 cases. Scand I Gastroenterol 1997;32:48-51.

39 Joshi AY, lyer VN, Hagan JB, et al. Incidence and temporal trends of primary immunodeficiency: a population-based cohort study. Mayo Clin Proc 2009:84:16-22.

40 Van Vollenhoven RF, Emery P, Bingham CO III, et al. Long-term safety of rituximab in rheumatoid arthritis: 9.5-year follow-up of the global clinical trial programme with a focus on adverse events of interest in RA patients. Ann Rheum Dis 2013;72:1496-502.

41 Mogensen TH, Bernth-Jensen JM, Petersen CC, et al. Common variable immunodeficiency unmasked by treatment of immune thrombocytopenic purpura with Rituximab. BMC Hematol 2013;3:4.

42 Diwakar L, Gorrie S, Richter A, et al. Does rituximab aggravate pre-existing hypogammaglobulinaemia? J Clin Pathol 2010;63:275-7.

43 Gottenberg JE, Ravaud P, Bardin T, et al. Risk factors for severe infections in patients with rheumatoid arthritis treated with Rituximab in the autolmmunity and Rituximab registry. Arthritis Rheum 2010;62:2625-32.

44 Puck JM. Laboratory technology for population-based screening for severe combined immunodeficiency in neonates: the winner is T-cell receptor excision circles. J Allergy Clin Immunol 2012;129:607-16. 